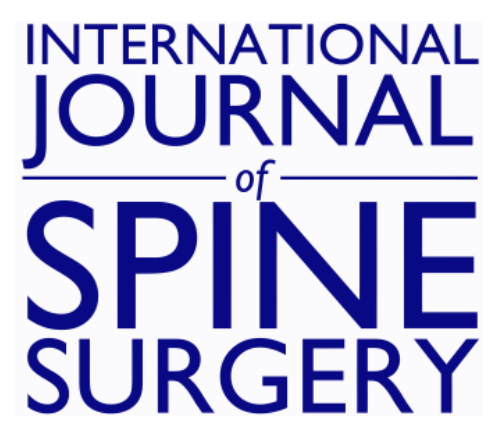

\title{
The Parallax Effect in the Evaluation of Range of Motion in Lumbar Total Disc Replacement
}

Joshua D. Auerbach, Surena Namdari, Andrew H. Milby, Andrew P. White, Sudheer C. Reddy, Baron S. Lonner and Richard A. Balderston

Int J Spine Surg 2008, 2 (4) 184-188

doi: https://doi.org/10.1016/SASJ-2008-0020-RR

http://ijssurgery.com/content/2/4/184

This information is current as of April 26, 2023.

Email Alerts Receive free email-alerts when new articles cite this article. Sign up at:

http://ijssurgery.com/alerts

The International Journal of Spine Surgery

2397 Waterbury Circle, Suite 1,

Aurora, IL 60504, Phone: +1-630-375-1432 


\title{
The Parallax Effect in the Evaluation of Range of Motion in Lumbar Total Disc Replacement
}

\author{
Joshua D. Auerbach, MD, ${ }^{a}$ Surena Namdari, MD, ${ }^{b}$ Andrew H. Milby, BA, ${ }^{c}$ Andrew P. White, MD, ${ }^{d}$ \\ Sudheer C. Reddy, MD, ${ }^{b}$ Baron S. Lonner, MD, ${ }^{e}$ and Richard A. Balderston, $M D^{f}$
}

\begin{abstract}

\section{Background}

Range of motion (ROM) has been shown to influence clinical outcomes of total disc replacement (TDR). While the parallax effect in image acquisition has been shown in the literature to influence the accuracy of a variety of measurements, this concept has not been investigated in the assessment of ROM analysis following TDR.
\end{abstract}

We performed an evaluation of the influence of radiograph beam angle on "by hand" and on "gold standard" flexionextension ROM measurements in lumbar total disc replacement. The purpose of this study is to determine (1) the influence of X-ray beam angle on index level angle (ILA) measurements in lumbar TDR using the keel method, and (2) whether the out-of-plane radiographic beam effects cause a difference between true and calculated range of motion.

\section{Methods}

Eight blinded orthopaedic surgeons used the keel method to calculate ROM measurements from radiographs of a flexible Sawbones model (Pacific Research Laboratories, Inc., Vashon, Washington) implanted with a ProDisc-L device (Synthes Spine, West Chester, Pennsylvania). Radiographs were obtained at beam angles of $0^{\circ}, 5^{\circ}, 10^{\circ}$, and $15^{\circ}$ in the sagittal plane from the device center. Calculations were compared to measurements obtained by a validated digitized software method (Quantitative Motion Analysis, QMA, Medical Metrics, Inc., Houston, Texas). Inter- and intraobserver precision and accuracy were determined.

\section{Results}

Compared with QMA, the radiographic keel method had an average error of $3.7^{\circ}$. No significant effect of variation in beam angle on interobserver precision $(\mathrm{N}=16, P=.92)$ or accuracy $(\mathrm{N}=16, P=0.86)$ or intraobserver precision $(\mathrm{N}=8, P=.09)$ or accuracy $(\mathrm{N}=8, P=0.07)$ of ROM measurements was identified. Repeat testing with QMA also revealed no effect of parallax and resulted in nearly identical ROM measurements.

\section{Conclusions}

Accuracy and precision of the keel method to determine ROM from index level angle measurements after TDR was not affected by increases in X-ray beam angles up to $15^{\circ}$ from the device center.

\section{Clinical Relevance}

Our study demonstrates that range of motion measurements are not influenced by parallax effect when using the keel method to determine index level angle measurements in lumbar total disc replacement.

Key Words: lumbar, disc replacement, parallax, range of motion. SAS Journal. December 2008;2:184-188. DOI: SASJ-2008-0020-RR

${ }^{a}$ The Department of Orthopaedic Surgery, Washington University School of Medicine, St. Louis, Missouri; ' $T$ The Department of Orthopaedic Surgery, University of Pennsylvania, Philadelphia, Pennsylvania; 'The University of Pennsylvania School of Medicine, Philadelphia, Pennsylvania; ${ }^{d}$ The Department of Orthopaedic Surgery, Beth Israel Deaconess Medical Center, Boston, Massachusetts; eDepartment of Orthopaedic Surgery, NYU-Hospital for Joint Diseases, New York, New York; f3B Orthopaedics, Pennsylvania Hospital, Philadelphia, Pennsylvania

Address correspondence to Joshua D. Auerbach, MD, Department of Orthopaedic Surgery, Washington University School of Medicine/ Barnes-Jewish Hospital, 660 South Euclid Avenue, Campus Box 8233, St. Louis, MO 63110 (email: auerspine@gmail.com)

This study was supported by Synthes Spine, West Chester, Pennsylvania. Dr. Balderston is a consultant to and has received research support from Synthes Spine. Dr. Auerbach has received research support from Synthes Spine.

Institutional Review Board approval was obtained for this study.

\section{INTRODUCTION}

One goal of lumbar total disc replacement (TDR) is to maintain or restore motion, in order to reduce the transfer of stresses to adjacent levels. ${ }^{1,2,3}$ Increased range of motion (ROM) of implanted lumbar TDR has recently been correlated with improved clinical outcomes. ${ }^{4,5,6}$ This correlation is partially responsible for the emergence of ROM measurements as pivotal radiographic outcome 
measures following lumbar TDR. As a result, there is interest in improving methods of ROM measurement and in identifying potential sources of ROM measurement error. ${ }^{5,7,8}$

In patients with preserved vertebral bony anatomy, flexionextension radiographs are taken, and the differences between index level angle (ILA) measurements taken in flexion and extension are utilized to calculate sagittal plane ROM. Generally, the ILA is measured as the angle subtended by the lines drawn along the cranial vertebral endplates. Though this same method is commonly used for patients with TDR, 2 potential confounders of accurate measurement exist-endplate removal in TDR and parallax effect during image acquisition. As the superior and inferior footplates of the implant replace the native endplates, an endplate no longer exists as a radiographic landmark for precise ILA measurement.

Second, parallax effect is a product of X-ray beam positioning during image acquisition. Specifically, parallax is created by aligning the X-ray beam at a fixed sagittal distance from the implant and then displacing the $\mathrm{X}$-ray source cranially to create an angle of the X-ray beam. While flexion-extension radiographs are typically performed with the beam centered on the mid lumbar spine, most TDR implants are at the L4-5 or L5-S1 segments. Since the implant is positioned caudal to the beam center, relative beam divergence may distort the radiographic landmarks used for flexion-extension ILA measurement. This effect can be exaggerated in large patients where the distance from the implant to the radiographic cassette is increased. The purpose of this study was to evaluate the influence of X-ray beam angle on measurement error in TDR. We hypothesized that parallax would not affect measurements of ROM when utilizing the keel method for ILA determination.

\section{MATERIAL AND METHODS Measurements}

A flexible lumbar Sawbones (Pacific Research Laboratories, Inc., Vashon, Washington) model was implanted with a large-sized ProDisc-L device (Synthes Spine, West Chester, Pennsylvania) using an $11^{\circ}$ inlay at the L4-5 level. Maximal lateral flexion and maximal extension radiographs were obtained using standard settings (96 kV X-ray beam, at $0.71 \mathrm{~mA}$ per second, with the object consistently placed $40 \mathrm{~cm}$ from the X-ray tube, onto $10 \times 12$-inch cassettes) with the beam $0^{\circ}, 5^{\circ}, 10^{\circ}$, and $15^{\circ}$ incident to the device center (Figures 1 and 2). This range of tested parallax angles was selected based on the normal parallax variation seen in the clinical setting.

In order to ensure maintenance of maximal motion implant flexion, a custom wooden spacer was placed in between the spinous processes and reinforced with elastic bands around the L4 and L5 transverse processes. Similarly, to ensure that maximal extension was achieved and maintained, multiple elastic bands were secured around the L4 and L5 transverse processes once the device was maximally extended.

\section{Figure 1.}

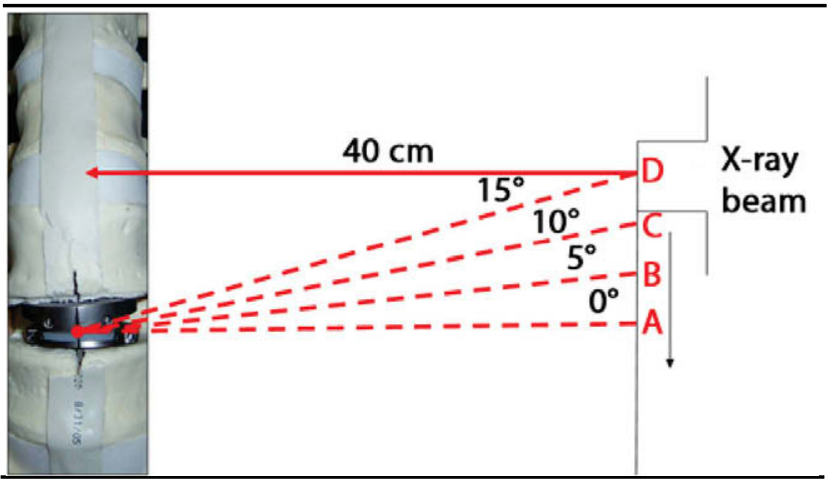

Figure 1 is a schematic of the technique used for variable $X$-ray beam acquisition. With the X-ray tube aiming parallel to the floor, the angle subtended between the X-ray beam and the device center was decreased from $15^{\circ}, 10^{\circ}, 5^{\circ}$, and $0^{\circ}$ in the sagittal plane by sequentially lowering the X-ray beam without changing the distance between the tube and the device (black arrow).

The exact measurements for vertical displacement (Vd) of the X-ray tube to produce angle $\theta$ were calculated trigonometrically using the following equation: $\mathrm{Vd}=(40$ $\mathrm{cm})(\tan \theta)$. The X-ray tube vertical displacements needed in order to achieve the above desired angles were as follows: $0^{\circ}-0 \mathrm{~cm} ; 5^{\circ}-3.5 \mathrm{~cm} ; 10^{\circ}-7.1 \mathrm{~cm} ; 15^{\circ}-10.7 \mathrm{~cm}$. The technique for generating variable $\mathrm{X}$-ray beam angles is detailed in Figure 1. A calibrated, digital meter on the $\mathrm{X}$-ray unit was used to verify angle coordinates given the fixed distance from the device center. The beam was placed directly in line with the device center using a digitized laser localizer, which confirmed that there was no variability in the anterior-posterior direction. Equipment setup was supervised by an experienced X-ray technician to ensure proper calibration and coordinate determination.

The flexion to extension ROM was calculated as the difference between the flexion ILA and the extension ILA for each pair. Comparative measurements for angular ROM were obtained using Quantitative Motion Analysis software (QMA, Medical Metrics, Inc., Houston, Texas), which uses digital superposition methods to assess intervertebral motion. QMA is a computer-assisted technique similar to the method of Penning et al., ${ }^{9}$ with patented modifications to optimize accuracy and reproducibility, and to circumvent parallax effects. The technique has been validated, with an average reported measurement error of $0.5^{\circ} .9,10,11$ Cobb angle measurements of all 8 images were also performed by 8 orthopaedic surgeons and residents on 2 separate occasions using a \#2 pencil and hinged goniometer (or Cobb ruler of the surgeon's choice) at least 1 week apart, with the image order randomized prior to each occasion. 
Participants were blinded to the purpose of the study and were instructed to use only the keel method for ILA measurement (Figure 2), as this method has recently been shown to be more precise than use of the metallic prosthesis endplate as a landmark reference. ${ }^{8}$

\section{Figure 2.}

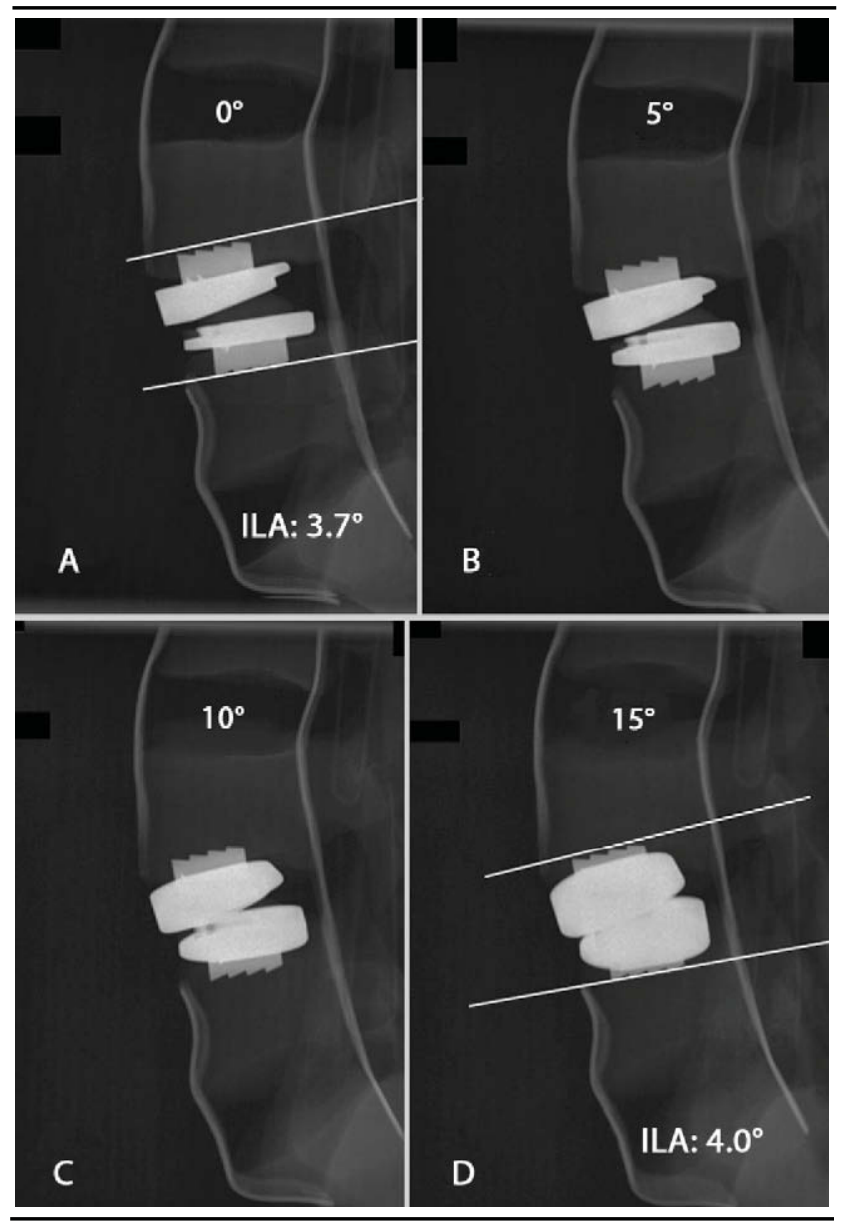

Sawbones model (Pacific Research Laboratories, Inc., Vashon, Washington) with lumbar total disc replacement (ProDisc- $L$, Synthes Spine, West Chester, Pennsylvania) implanted at the L4-5 interbody space in flexion. With the X-ray beam in the neutral position (A), the edges of the endplates are clearly identifiable. With increasing superior movement of the X-ray beam at $5^{\circ}(B), 10^{\circ}(C)$, and $15^{\circ}(D)$, notice the increasingly distorted projection of the footplates. The keel method, using lines drawn along the superior and inferior aspects of the keels, was used to determine Cobb measurements from which range of measurements were calculated. ${ }^{8} \mathrm{~A}$ comparison of ( $\mathrm{A}$ : neutral beam angle) and ( $D: 15^{\circ}$ beam angle) reveals a difference of only $0.3^{\circ}$ despite an X-ray beam angle of $15^{\circ}$.

ILA: index level angle

\section{Statistical Analysis}

The effect of beam angle on both inter- and intraobserver precision and accuracy was evaluated. Mean ROM and standard deviation were computed at each beam angle. Error terms for each observation were calculated by subtracting participants' ROM measurements from the corresponding gold standard values from the QMA software, allowing for the computation of mean error and standard deviation at each beam angle. Interobserver precision was assessed by comparing the distributions of ROM measurements at each of the 4 beam angles using 1 -way analysis of variance (ANOVA). The distributions of the absolute values of these error terms were analyzed with 1-way ANOVA to determine the effect of beam angle on interobserver accuracy. The absolute values of the differences between the participants' first and second measurements were calculated, and the distributions at each of the beam angles were compared with the Friedman rank sum test to assess intraobserver precision. The effects on intraobserver accuracy were determined by comparing the distributions of the differences in error between participants' first and second observations at each of the beam angles with the Friedman rank sum test. Statistical analysis was performed using Statistical Package for the Social Sciences (SPSS, Chicago, Illinois), version 15.0, with $P$ values less than .05 considered significant.

\section{RESULTS}

At beam angles $0^{\circ}, 5^{\circ}, 10^{\circ}$, and $15^{\circ}$ the QMA "gold standard" method yielded ROM values of $20.5^{\circ}, 20.6^{\circ}$, $20.3^{\circ}$, and $20.9^{\circ}$, respectively. A repeat analysis of the images was conducted 3 months later. The results from the second analysis were almost identical to the first and yielded ROM values of $20.4^{\circ}, 20.1^{\circ}, 20.3^{\circ}$, and $20.7^{\circ}$. In contrast, the "by hand" mean ROM values among the 8 observers were $24.7 \pm 4.6^{\circ}, 23.8 \pm 3.4^{\circ}, 24.1 \pm 3.3^{\circ}$, and $24.2 \pm 3.9^{\circ}$ (Table 1), yielding mean error terms from the gold standard of $4.3 \pm 4.6^{\circ}, 3.2 \pm 3.3^{\circ}, 3.80 \pm 3.3^{\circ}$, and $3.3 \pm 3.9^{\circ}$, respectively. No significant effect on either precision $(\mathrm{N}=16, P=.92)$ or accuracy $(\mathrm{N}=16, P=$ .86) of the ROM measurements among the observers was identified with variation in beam angle (Table 1, Figure $3)$. No significant effect on intraobserver precision $(\mathrm{N}=$ $8, P=.09)$ or accuracy $(\mathrm{N}=8, P=.07)$ was observed with variation in beam angle (Table 1 , Figure 4$)$.

Table 1. Inter- and Intraobserver Precision and Accuracy of Radiographic Range of Motion Measurement Using the Keel Method at Increasing Beam Angles

\begin{tabular}{|c|c|c|c|c|c|c|c|}
\hline Comparison & Calculation & $\mathbf{n}$ & $0^{\circ}$ & $5^{\circ}$ & $10^{\circ}$ & $15^{\circ}$ & $\bar{P}$ \\
\hline Interobserver precision $^{a}$ & ROM observations 1 and 2 combined & 16 & $24.7 \pm 4.6$ & $23.8 \pm 3.4$ & $24.1 \pm 3.3$ & $24.2 \pm 3.9$ & .92 \\
\hline Intraobserver accuracy ${ }^{a}$ & ROM error observations 1 and 2 combined & 16 & $4.3 \pm 4.6$ & $3.2 \pm 3.3$ & $3.8 \pm 3.3$ & $3.3 \pm 3.9$ & .86 \\
\hline Interobserver precision ${ }^{b}$ & ROM observation 1 - ROM observation 2 & 8 & $2.0(0.0-10.0)$ & $3.0(1.0-10.0)$ & $1.5(0.0-10.0)$ & $1.0(0.0-3.0)$ & .09 \\
\hline Intraobserver accuracy ${ }^{b}$ & ROM error observation 1 - ROM error observation 2 & 8 & $2.0(0.0-10.0)$ & $3.0(0.2-10.0)$ & $1.5(0.0-9.4)$ & $1.0(0.0-3.0)$ & .07 \\
\hline
\end{tabular}


Figure 3.

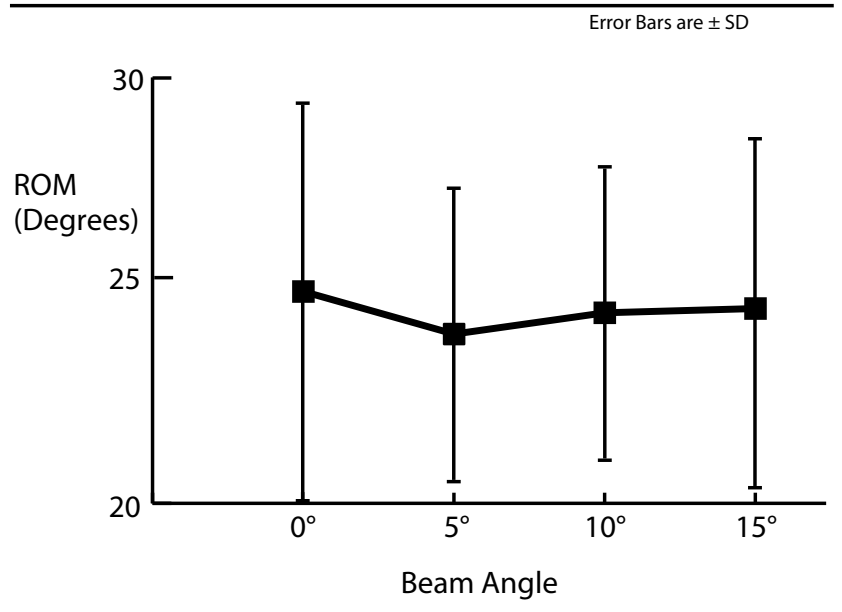

Minimal variation in mean ROM measurement as beam angle increases.

Figure 4.

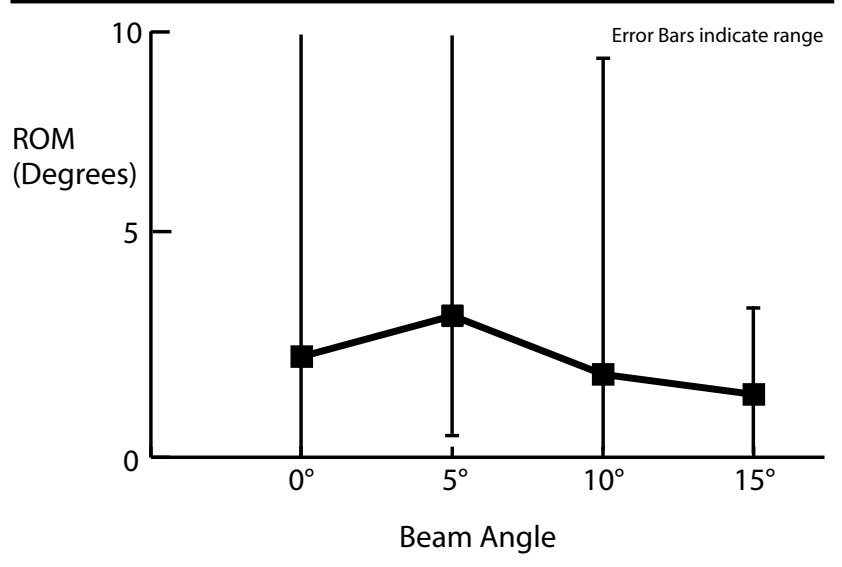

Minimal variation in median intra-observer ROM error as beam angle increases.

\section{DISCUSSION}

Parallax is defined as the apparent displacement or the difference in apparent direction of an object as seen from 2 different points not on a straight line with the object. ${ }^{12}$ In image acquisition, the position of the center of the Xray beam determines the position of the observer. This concept has been investigated in the medical literature in the radiographic assessment of short trachea in infants, ${ }^{13}$ inferior vena cava filter position,,${ }^{14}$ determination of joint space width in the hip, ${ }^{15}$ and in the quantification of slip measurement in spondylolisthesis. ${ }^{16}$ If the film or the vertebral body are tilted or off-center from the central beam, the projection of the contours of vertebral bodies may become distorted (ie, induce parallax), which can result in erroneous measurements. ${ }^{17}$ With increasing focus on the importance of ROM in TDR, the methods of ROM measurement may require refinement to ensure accuracy of device performance assessment. $5,7,8,17,18,19$
The results of this study demonstrate that variability in Xray beam angle in the range of $0-15^{\circ}$ does not significantly alter inter- and intraobserver reliability of ROM calculation in TDR. This study supports our hypothesis that the keel method of determining ILA and ROM measurements in TDR is not influenced by parallax effect. It should also be noted that the QMA technique was not sensitive to parallax, and repeat testing at 3 months revealed nearly identical values for ROM. Although out-of-plane effects do not affect ROM assessment in TDR using the keel method, future study should evaluate the effect of parallax in nonkeeled devices, the endplate method for ILA measurements, and an assessment of parallax in multiple planes (ie, axial rotation commonly seen with patient twisting).

This manuscript was submitted August 17, 2008, and accepted for publication November 19, 2008.

The authors would like to thank the following for their contributions to the manuscript: Nicholas D. Wharton, Miltiadis H. Zgonis, Carrie M. Ballester, Amer F. Samdani, Reginald Fayssoux, Ejovi Ughwanogho, and Okechukwu Anakwenze.

\section{REFERENCES}

1. Auerbach JD, Wills BP, McIntosh TC, Balderston RA. Evaluation of spinal kinematics following lumbar total disc replacement and circumferential fusion using in vivo fluoroscopy. Spine. 2007;32(5):527-536.

2. Cunningham BW, Gordon JD, Dmitriev AE, Hu N, McAfee PC. Biomechanical evaluation of total disc replacement arthroplasty: an in vitro human cadaveric model. Spine. 2003;28(20):S110-117.

3. O'Leary P, Nicolakis M, Lorenz MA, et al. Response of Charite total disc replacement under physiologic loads: prosthesis component motion patterns. Spine J. 2005;5(6):590-599.

4. McAfee PC, Cunningham B, Holsapple G, et al. A prospective, randomized, multicenter Food and Drug Administration investigational device exemption study of lumbar total disc replacement with the CHARITE artificial disc versus lumbar fusion: part II: evaluation of radiographic outcomes and correlation of surgical technique accuracy with clinical outcomes. Spine. 2005;30(14):1576-1583; discussion E388-390.

5. Huang RC, Girardi FP, Cammisa FP Jr, Lim MR, Tropiano P, Marnay T. Correlation between range of motion and outcome after lumbar total disc replacement: 8.6-year follow-up. Spine. 2005;30(12):1407-1411.

6. Chung SS, Lee CS, Kang CS. Lumbar total disc replacement using ProDisc II: a prospective study with a 2-year minimum follow-up. J Spinal Disord Tech. 2006;19(6):411-415. 
7. Cakir B, Richter M, Puhl W, Schmidt R. Reliability of motion measurements after total disc replacement: the spike and the fin method. Eur Spine J. 2006;15(2):165173.

8. Lim MR, Girardi FP, Zhang K, Huang RC, Peterson MG, Cammisa FP Jr. Measurement of total disc replacement radiographic range of motion: a comparison of two techniques. J Spinal Disord Tech. 2005;18(3):252-256.

9. Penning L, Irwan R, Oudkerk M. Measurement of angular and linear segmental lumbar spine flexionextension motion by means of image registration. Eur Spine J. 2005;14(2):163-170.

10. Subramanian N, Reitman CA, Nguyen L, Hipp JA. Radiographic assessment and quantitative motion analysis of the cervical spine after serial sectioning of the anterior ligamentous structures. Spine. 2007;32(5):518-526.

11. Zhao K, Yang C, Zhao C, An KN. Assessment of noninvasive intervertebral motion measurements in the lumbar spine. J Biomech. 2005;38(9):1943-19

12. Merriam-Webster Medical Dictionary. Parallax. Merriam-Webster Medical Dictionary. http://www2. merriam-webster.com/cgi-bin/mwmednlm\&book=M edical\&va=parallax. Accessed October 15, 2008.

13. Wells TR, Landing BH, Padua EM. The question of parallax-effect on radiographic assessment of short trachea in infants and children. Pediatr Radiol. 1991;21(7):490-493.

14. Wolfer GK Jr, Taylor FC, Smith DC. Quantification of the effects of respiration and parallax on inferior vena caval filter position. J Vasc Interv Radiol. 1994;5(2):357-360.

15. Goker B, Sancak A, Haznedaroglu S, Arac M, Block JA. The effects of minor hip flexion, abduction or adduction and X-ray beam angle on the radiographic joint space width of the hip. Osteoarthritis Cartilage. 2005;13(5):379-386.

16. Wall MS, Oppenheim WL. Measurement error of spondylolisthesis as a function of radiographic beam angle. J Pediatr Orthop. 1995;15(2):193-198.

17. Frobin W, Brinckmann P, Leivseth G, Biggemann M, Reikeras O. Precision measurement of segmental motion from flexion-extension radiographs of the lumbar spine. Clin Biomech (Bristol, Avon). 1996;11(8):457-465.

18. Kosmopoulos V, McManus J, Schizas C. Consequences of patient position in the radiographic measurement of artificial disc replacement angles. Eur Spine J. 2008; 17(1):30-35.
19. Petilon J, Hardenbrook M, Sukovich W. The effect of parallax on intraoperative positioning of the Charite artificial disc. J Spinal Disord Tech. 2008;21(6):422429. 